\title{
Gilles HOULE (1947-2006)
}

Sociologue, Université de Montréal

(1997)

\section{"La sociologie comme science du vivant: l'approche biographique”}

Un document produit en version numérique par Jean-Marie Tremblay, bénévole, professeur de sociologie au Cégep de Chicoutimi

Courriel: jean-marie tremblay@uqac.ca

Site web pédagogique : http://www.uqac.ca/jmt-sociologue/

Dans le cadre de: "Les classiques des sciences sociales" Une bibliothèque numérique fondée et dirigée par Jean-Marie Tremblay, professeur de sociologie au Cégep de Chicoutimi Site web: http://classiques.uqac.ca/

Une collection développée en collaboration avec la Bibliothèque Paul-Émile-Boulet de l'Université du Québec à Chicoutimi

Site web: http://bibliotheque.uqac.ca/ 
Cette édition électronique a été réalisée par Jean-Marie Tremblay, bénévole, professeur de sociologie au Cégep de Chicoutimi à partir de :

Gilles Houle (1947-2006)

“La sociologie comme science du vivant : l'approche biographique”.

Un article publié dans l'ouvrage sous la direction de Poupart, Deslauriers, Groulx, Laperrière, Mayer, Pires [Groupe de recherche interdisciplinaire sur les méthodes qualitatives], La recherche qualitative. Enjeux épistémologiques et méthodologique, pp. 273-289. Montréal : Gaëtan Morin, Éditeur, 1997, 405 pp.

M Gilles Houle, sociologue (décédé) de l’Université de Montréal, nous a accordé le 8 février 2004 son autorisation de diffuser électroniquement toutes ses oeuvres.

Polices de caractères utilisée :

Pour le texte: Times New Roman, 14 points.

Pour les citations : Times New Roman, 12 points.

Pour les notes de bas de page : Times New Roman, 12 points.

Édition électronique réalisée avec le traitement de textes Microsoft Word 2004 pour Macintosh.

Mise en page sur papier format

LETTRE (US letter), 8.5'’ x 11'’)

Édition numérique réalisée le 29 juin 2007 à Chicoutimi, Ville de Saguenay, province de Québec, Canada. 


\section{Gilles Houle (1947-2006)}

\section{"La sociologie comme science du vivant: l'approche biographique”}

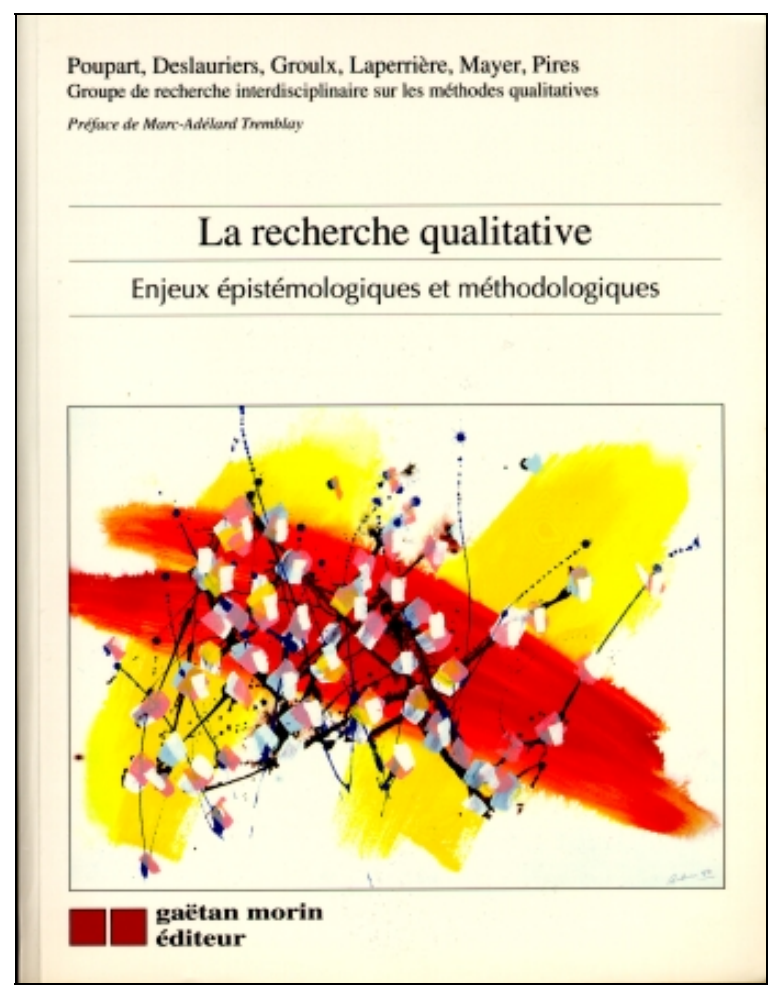

Un article publié dans l'ouvrage sous la direction de Poupart, Deslauriers, Groulx, Laperrière, Mayer, Pires [Groupe de recherche interdisciplinaire sur les méthodes qualitatives], La recherche qualitative. Enjeux épistémologiques et méthodologique, pp. 273-289. Montréal : Gaëtan Morin, Éditeur, 1997, 405 pp. 


\section{Table des matières}

$\underline{\text { Introduction }}$

LE PROBLÈME DES DÉFINITIONS

UN NOUVEL ESPACE THÉORIQUE

HISTOIRE OU RÉCIT DE VIE : UNE HISTOIRE DE CAS

Les histoires de vie comme base de données

Les histoires de vie comme connaissance

Un modèle concret

De l'analyse

CONCLUSION

Bibliographie 
Gilles Houle (1947-2006)

"La sociologie comme science du vivant : l'approche biographique".

Un article publié dans l'ouvrage sous la direction de Poupart, Deslauriers, Groulx, Laperrière, Mayer, Pires [Groupe de recherche interdisciplinaire sur les méthodes qualitatives], La recherche qualitative. Enjeux épistémologiques et méthodologique, pp. 273-289. Montréal : Gaëtan Morin, Éditeur, 1997, 405 pp.

\section{Introduction}

$\underline{\text { Retour à la table des matières }}$

La redécouverte, au début des années 70, des histoires ou récits de vie aura été d'une importance considérable pour la sociologie (voir un classique sur le sujet : Bertaux, 1980, 1981). Si l'usage que l'on en fit fut multiple et souvent problématique, il n'empêche que la célébration en quelque sorte de ce type de données aura tout de même permis de mettre la sociologie face à elle-même et, notamment, aux exigences inhérentes à toute démarche explicative. Si sciences sociales il y a, ces exigences valent pour toutes les disciplines, quel que soit leur objet, et il n'est pas de raison que la sociologie échappe à de pareilles interrogations. En somme, après avoir congédié le sujet par trop encombrant dans l'analyse des processus sociaux, voilà que l'on applaudit maintenant son retour (Touraine, 1984), le retour d'un sujet qui en fait n'était jamais parti. Que s'est-il donc passé et que peut nous apprendre cette redécouverte de ce point de vue? 
Il n'est plus nécessaire de faire la démonstration de l'importance des histoires ou récits de vie dans les sciences humaines; non plus de faire le dénombrement des recherches qui auront privilégié ce type de matériau, qui le privilégient ou comptent encore le privilégier. Leur nombre est en fait à la mesure même des attentes qu'aura suscitées cette nouvelle «méthode » biographique. Or le renouvellement paradigmatique annoncé s'est heurté à des problèmes théoriques et méthodologiques dont on aura tout au plus aperçu la complexité. Cette complexité est pourtant bien réelle et pourrait résumer l'immense intérêt de cette redécouverte de l'école de Chicago et des enjeux théoriques, méthodologiques aussi bien qu'épistémologiques qui ont été alors "approchés » (Bachelard, 1968). Ni technique, ni méthode, ni théorie (Houle, 1986), ce matériau appelle pourtant une approche renouvelée en sociologie, mais surtout recèle par ses qualités propres une valeur heuristique considérable. Il importe de définir ces enjeux, car l'impossibilité virtuelle de résoudre les problèmes soulevés, de surmonter les obstacles épistémologiques rencontrés, pourrait bien, comme ce fut le cas à Chicago, nous faire découvrir une deuxième fois les vertus du questionnaire, de la ou des méthodes quantitatives, dont la critique, dira-t-on, aura été trop sévère. Et pourquoi pas ? Il est à vrai dire impossible de dresser un bilan ${ }^{1}$ de l'ensemble de ces recherches; je m'emploierai plutôt à relever les problèmes que pose l'analyse des histoires de vie pour tenter de recenser ces enjeux " approchés », d'en donner une première définition ne serait-ce que pour m'assurer qu'ils auront été notés avant que ce matériau retombe dans l'oubli et qu'après avoir critiqué aussi sévèrement l'usage du questionnaire, il ne reste plus qu'à le réinventer. Peut-on éviter ce retour du pendule ?

Si ce n’est pas le lieu d'expliciter ici la perspective adoptée, je rappelle pour mémoire que je récuse l'opposition classiquement affirmée

1 La première bibliographie publiée en 1980 des travaux se fondant sur les histoires de vie comptait quatre pages, en petits caractères (voir Bertaux, 1981 : 221-225). La dernière en date que je connaisse, publiée en 1995, compte 37 pages, en petits caractères aussi (voir Simeoni et Diani, 1995 : 221-258). 
entre les méthodes qualitatives et quantitatives (Houle, 1982) au nom précisément de ce que serait une méthodologie générale en sciences sociales. Il n'est à vrai dire pas beaucoup d'exemples dans l'histoire des sciences où le statut d'une méthode est défini moins par l'objet de la discipline que par la nature des données à analyser. Le débat qualitatif/quantitatif, encore que de rigueur, paraît en voie d'être dépassé, là où des chiffres et des lettres justifient d'emblée des méthodes techniquement différentes dans une démarche explicative qui satisfasse néanmoins les mêmes exigences de rigueur (Pires, 1994).

Je n'ai pas par ailleurs la naïveté de croire que cette opposition n'avait pas, n'a pas quelque prégnance tant il est vrai que les « qualitatifs » se doivent, voire sont mis en demeure, d'exhiber leurs lettres de créance méthodologique, c'est-à-dire de démontrer de quelle scientificité ils sont capables; et l'on sait bien d'où vient, qui pose cette question. De manière plus précise, j'aborderai ces questions dans le prolongement de travaux connus, d'une tradition épistémologique où, de Bachelard à Granger, je me situerai au sein du « front commun des travailleurs de la preuve " (Bachelard, 1949) avec le premier, sans oublier avec le second que l'on a beau « être l'ami des formes, l'on reste fils de la terre » (Granger, 1968). Cette question de la scientificité est pourtant posée et vaut pour tout le monde dans la mise en cause de tous les positivismes, qu'ils soient d'ailleurs qualitatifs ou bien quantitatifs.

\section{LE PROBLÈME DES DÉFINITIONS}

$\underline{\text { Retour à la table des matières }}$

La redécouverte des histoires ou récits de vie, pour importante qu'elle soit, reste cependant étonnante, car ce n'était pas là le matériau privilégié par Thomas et Znaniecki, ce n'est pas là que réside l'originalité de l'école de Chicago. La référence obligée au Polish Peasant (Thomas et Znaniecki, 1958) met en évidence l'usage de correspon- 
dances beaucoup plus que de récits de vie dans la recherche alors effectuée. L'usage des récits de vie était second, complémentaire, pour tout dire, tant les correspondances étaient considérées comme un matériaux parfait, et il convient de le souligner. Nous y reviendrons. Ce sont pourtant des histoires ou des récits que l'on se rappellera. Une sociologie de la sociologie pourrait sans doute vérifier qu'il n'y a rien là que de normal ; cette redécouverte s'insère dans la mouvance plus générale et plus récente de la découverte des " écritures du moi » pour reprendre ici l'expression de Gusdorf (1990) ; les biographies et autobiographies n'ont en effet jamais été aussi populaires. Les sociologues en auront aussi procédé, comme tous les autres, oubliant au passage le journal intime également privilégié par les chercheurs de l'école de Chicago. Reste alors à découvrir le chemin qui, pour les sociologues, pourrait mener des écritures du «moi » aux écritures du «soi » (Mead, 1934), car pour individuels que soient les récits, correspondances ou journaux intimes, ces discours donnent accès à une pratique qui est aussi sociale (Houle, 1993b).

Cette redécouverte des histoires de vie est à la mesure des sciences humaines, c'est-à-dire polysémique, par ce que sont les sciences humaines prises une à une, mais aussi par la fluidité des objets de recherche au sein de chacune de ces disciplines. On recueillera donc selon le cas des histoires de vie, des récits de vie, des récits de pratique en sociologie notamment ; les historiens plus modestement feront de l'histoire orale ; les littéraires s'intéresseront pour leur part à l'autobiographie orale. Les psychanalystes et psychothérapeutes d'étiquettes diverses continuent leur travail sans plus, comme s'il s'agissait d'un non-lieu. Du récit de vie au récit de la vie, la table est mise pour d'innombrables recherches que l'on ne peut expliquer que par la variété des objets de recherche qui leur sont dévolus, et ce dans des acceptions les plus diverses qui soient. Les historiens font de l'histoire, les littéraires de la littérature et les sociologues de la sociologie, bien sûr ; mais l'horizon tracé depuis le récit de vie en fait jusqu'au récit de la vie permet de poser un regard neuf sur l'objet de chacune de ces disciplines aussi bien que sur leur visée. Thompson (1978) aura posé la 
question en histoire : Qui fait l'histoire ? De qui écrit-on l'histoire ? Comment écrit-on l'histoire ? Qu'est-ce donc que la littérature ? Plus justement, qu'est-elle devenue ? Pour la sociologie, Ferrarotti résume ainsi la situation actuelle :

Au-delà de l'univocité présumée de la raison, le sociologue devra tendre l'oreille aux motivations qui se manifestent à travers les gestes, les expressions faciales, les inflexions de voix, dans tout ce magma qui constitue le modèle «mou » du vécu, le rapport au corps qui ne parvient pas à vivre et, à la limite, pas même à entrer dans la logique cartésienne de l'écriture. Il est une polysémie du quotidien à l'exploration de laquelle le sociologue est tenu, qui constitue même son champ d'analyse privilégié et qui échappe irrémédiablement au schématisme du scientifique « dur ». Dans cette perspective et en ce sens, la crise actuelle de la sociologie, la crise de sa propre image et de sa méthode, est une crise salutaire. (Ferrarotti, 1989 2.)

En effet, les sociologues, pour célébrer le retour du sujet dans la redécouverte de son vécu, doivent maintenant faire face à cette crise, c'est-à-dire prendre la mesure des questions théoriques et méthodologiques dès lors soulevées. Et notamment celle-ci : le récit ou l'histoire d'une vie ne renvoie pas qu'au vécu d'un sujet, il est aussi et dans le même temps le récit ou l'histoire de la vie en société. Cette question est fondamentale dans la mesure même où cela nous renvoie aux fondements de la discipline sociologique. Les histoires de vie nous racontent en fait l'histoire de la vie en société et nous obligent à redécouvrir aussi ce qui est bien l'objet ultime de la sociologie, le vivant. Faut-il souligner davantage ce que pourrait être le pouvoir heuristique de ce matériau ? Quel statut théorique, épistémologique lui donner ? Sur cette base, quelle démarche d'analyse pourrait s'en trouver alors autorisée ?

2 Je remercie Isabelle Perrault pour cette traduction. 


\section{UN NOUVEL ESPACE THÉORIQUE}

$\underline{\text { Retour à la table des matières }}$

L'enjeu est celui-ci : ce matériau, ces données, les propos tenus dans ces histoires ou récits valent. Dans les années 70, le sujet n'est pas encore tout à fait de retour, c'est-à-dire reconnu tel, mais sa parole vaut. Le sujet, ainsi dénommé, ne serait plus aliéné, n'occulterait plus la réalité, victime de quelque idéologie dominante qui l'interpellerait en sujet. Giddens (1987) écrira qu'il est compétent ; mais sa compétence ici reconnue est ethnométhodologique, précisons-le. Plus simplement, sa parole vaut, le sujet parle et ne dit plus de bêtises que le sociologue viendrait corriger dans la différenciation d'une sociologie spontanée et d'une sociologie savante. Notre objet parle, écrira Bourdieu. En effet, et cette parole fait sens, y compris en l'absence du sens que le sociologue voudrait bien lui conférer. On souhaitera jusqu'à pénétrer son intimité pour y trouver, si ce n'est des secrets, du moins le secret de ce que fut sa vie, de ce que serait la vie (Brunet et Gagnon, 1993).

Le regard est à vrai dire nouveau. Pour avoir participé à ce retour, j'ai connu cette première période où la richesse du récit -du vécu, a-ton aussi écrit - était telle qu'il suffisait de le publier avec une note introductive au besoin ; tout était là, au lecteur d'apprécier cette sociologie in vivo en somme. La deuxième aussi, où tout est là, mais encore faut-il pouvoir lire cette sociologie du vécu dans le récit. Cette difficulté reconnue, le sociologue s'y attaquera en dégageant la sociologie qui serait encastrée en quelque sorte dans l'histoire de vie. Et une troisième période ${ }^{3}$, enfin, où les questionnements commencent à traver-

3 La redécouverte des histoires ou récits de vie, leur usage remonte au début des années 70. Les trois périodes ici décrites se suivent sans qu'il soit possible de 
ser une variété d'analyses qui défient toute typologie mais donnent néanmoins la mesure de ce courant intellectuel. Un premier bilan des deux premières périodes laisse songeur quant aux analyses alors réalisées qui consistaient soit à s'identifier à l'auteur du récit, soit à lui conférer une identité qui n'était pas la sienne, autrement dit à faire du sujet un sociologue malgré lui. D'où les questionnements qui se font jour, dans la troisième période, sur le statut épistémologique du projet du chercheur, notamment en ce qui concerne la prise en compte et la description de la relation établie entre le sociologue et le sujet, plus généralement entre l'observateur et l'observé.

La question est dès lors celle-ci : quel statut théorique reconnaître au sujet, qui donne un fondement légitime, c'est-à-dire éthique et scientifique, à l'entreprise de recherche ? La question n'est pas nouvelle, mais le regard posé autorise une nouvelle réponse, justifie d'autres choix que le congédiement. La question est de taille et constitue l'enjeu théorique majeur que fait surgir le recours aux histoires de vie, mais aussi à la correspondance et aux journaux intimes : le statut théorique donné au sujet, à la subjectivité, au sens qu'il donne à la vie, c'est-à-dire à la connaissance qui est sienne, scelle le sort qui lui est réservé depuis son congédiement jusqu'à son retour qui reste pourtant problématique dans l'état actuel des choses. Et ce n'est pas une mauvaise plaisanterie que d'affirmer qu'il peut encore y perdre jusqu'à sa camisole. Nier le sujet, nier la relation qui doit s'établir entre le chercheur et l'historien de sa vie interdit, faut-il le préciser, tout espace théorique. Le chemin parcouru relativement à la description de la relation de l'observateur avec l'observé dans l'usage des histoires de vie permet de poser dans des termes nouveaux la question de leur identité respective, de l'altérité, mais aussi du statut du savoir de chacun tant il est vrai que leur projet n'est pas le même.

vraiment les dater tant elles se suivent et se recoupent selon les lieux et les projets de recherche, selon le moment de ladite redécouverte. 
Les correspondances analysées à Chicago permettent d'apprécier au mieux ce problème. La correspondance entretenue par les immigrants polonais et leurs familles ou amis était la mise en forme première, immédiate, de la vie à Chicago, des problèmes, des joies et des peines alors racontés, discutés, expliqués, et ce du point de vue même de ceux-là qui les vivaient. Ce matériau était " parfait », cette parole valait, faisait sens parce qu'elle est connaissance. Ce savoir des immigrants sur la vie des immigrants est spontané et n'a d'ailleurs que faire de la sociologie qui, rappelons-le, a d'autres visées. Voilà donc la vie des immigrants telle qu'elle apparaît dans l'intimité de leur vie. Les correspondances, les récits ou histoires de vie ne sont pas une simple collection d'informations à repérer aux fins de la recherche, d'une recherche en particulier ; ils sont le résultat d'une mise en forme suivant des modalités particulières à définir et à analyser en tant que telles, que ce soit du point de vue de la sociologie, de l'histoire, de la psychologie, etc. Ce savoir vaut et ne justifie en rien sa disqualification dans l'établissement d'un savoir sociologique ou autre ; il appelle une sociologie non pas du " vécu », mais du vivant tant il est vrai, faut-il le rappeler, que l'objet de notre discipline relève du vivant, que ce savoir, que le savoir premier du sociologue et la pluralité des savoirs dont procède le sociologue sont la base même de son travail et l'horizon radical de toute visée scientifique qui en définit la légitimité.

Sur la base de son expérience particulière, le sociologue travaille à partir d'expériences singulières à l'élaboration d'un savoir sociologique. Or comment assurer le passage de cette expérience, de ces expériences, c'est-à-dire de ces savoirs, au savoir sociologique, et dans quelles conditions ? Voilà le problème théorique que l'usage des correspondances ou des récits de vie permet d'apercevoir. C'est l'espace de l'observateur et de l'observé dans la relation construite ou à construire qui viendra définir, délimiter cet espace jusqu'à sa négation selon le statut qui sera accordé à Fun mais surtout à l'autre dans cette relation, comme nous l'avons vu plus haut. Inférer que le sujet racontant sa vie est sociologue ou qu'il fait de la sociologie sans le savoir, c'est nier à proprement parler son identité aussi bien que celle du 
sociologue qui a bien mal pris la mesure de son travail. Après avoir congédié le sujet dont il salue maintenant le retour, fallait-il vraiment qu'il se congédie lui-même ? Si ce fut le cas pendant un instant, il est permis maintenant de voir les choses autrement. Cet espace est aussi celui de l'intervieweur et de l'interviewé dans l'entretien à réaliser ; de l'analyste et de celui qui se raconte dans l'explication recherchée. Cet espace est à vrai dire proprement clinique et Jacques Ardoino l'a très bien décrit :

Est donc proprement clinique, aujourd'hui, ce qui veut appréhender le sujet (individuel et/ou collectif) à travers un système de relations, constitué en dispositif, c'est-à-dire au sein duquel le praticien, ou le chercheur, comme leurs partenaires, se reconnaissent effectivement impliqués, qu'il s'agisse de viser l'évolution, le développement, la transformation d'un tel sujet ou la production de connaissances, en soi comme pour lui ou pour nous. (Ardoino, 1989 : 65.)

Le renouveau de l'approche clinique en sociologie 4 ainsi conjugué à ces usages aura permis de redécouvrir en fait ce qui était déjà là dans la tradition sociologique, aussi bien que dans la tradition anthropologique. Dans la biographie qu'elle a consacrée à ses parents Margaret Mead et Gregory Bateson, Marie-Catherine Bateson nous donne une description tout à fait saisissante de leur façon de travailler, de ces conditions du travail théorique qui préparent ce passage du sens commun au sens sociologique :

Margaret ainsi que Gregory développèrent un style : ils collectaient des données d'observation dans l'espoir que, quelque riches et déroutantes qu'elles puissent paraître de prime abord, ils parviendraient à localiser des points de repère quand les choses "prendraient un sens » et se mettraient en place. Dans la recherche de tels moments de pénétration cognitive, ils examinaient les points de convergence au sein de la culture étudiée et aussi les lieux de réaction personnelle.

4 Enriquez et autres, 1993 ; De Gaulejac et Roy, 1993. Ce renouveau se conjugue sans doute avec la redécouverte des histoires de vie. Sa disparition reste mystérieuse à la différence de la découverte des histoires de vie, fort bien expliquée par Pires (1994). On pourra lire également le texte de Wirth (1931). 
Cette démarche est d'ordre esthétique : elle consiste à capter la résonance entre l'intérieur et l'extérieur, l'écho qui focalisera l'attention. Le poète fonctionne de la même façon quand le galbe d'une feuille évoque pour lui le caractère poignant d'un moment passé. Le praticien engagé dans une thérapie fonctionne également de cette façon-là ; il est animé d'un mouvement pendulaire entre l'impératif de sa tâche d'autoconnaissance et celle de compréhension de son patient, et il sait que, sans cette double intuition cognitive, la connaissance n'existe pas. Réellement, j'ai toujours conçu cet effort de prise de conscience et d'utilisation systématique des processus internes selon les termes qu'a employés Erik Erikson pour décrire la méthode clinique comme une "subjectivité disciplinée ». (Bateson, 1989 : 205.)

Cet espace théorique est désormais ouvert, et nous avons une définition théorique, grâce à Jacques Ardoino, ainsi qu'une description saisissante, grâce à Marie-Christine Bateson, du travail en train de se faire. La description de cette «subjectivité disciplinée » permet d'apercevoir la spécificité de cette démarche qui « renvoie, écrira Michel Foucault, aux conditions non verbales à partir de quoi il faut parler : la structure commune qui découpe et articule ce qui se voit et ce qui se dit » (Foucault, $1972: \mathrm{xv}$ ). Il soulignera à ce titre la retenue du discours clinique qui nous permet maintenant de parler de cette structure commune à l'observateur et à l'observé qui délimite cet espace qu'il reste pourtant à construire.

Ces nouvelles conditions non verbales à partir de quoi il faut parler sont celles d'un observateur dont les interrogations ne sont pas seulement théoriques et méthodologiques, mais aussi épistémologiques. Son implication, le statut de l'observé et la nécessité de la distance à construire dans une intimité partagée délimitent un nouvel espace et il faudra au sociologue sans doute faire preuve d'une subjectivité en effet fort disciplinée dans ces sciences où, il faut bien le reconnaître, la retenue du discours n'est pas de rigueur. Reste en somme à expliciter ou à définir les règles de cette discipline à laquelle devrait se plier la subjectivité du chercheur dans l'objectivité à construire (Devereux, 1980). Il reste en somme à définir le régime auquel le sociologue doit se soumettre. 
Grâce à Marie-Christine Bateson, nous avons une première idée de ce que devraient être les conditions de travail du sociologue. Reste à voir quelles sont les règles qui devraient régir ce travail dans le passage d'une subjectivité 5 , de subjectivités, à l'objectivité. Ces règles sont à l'évidence théoriques et méthodologiques, mais il n'en demeure pas moins qu'il faut souligner l'apport déterminant de la démarche clinique dans la définition de l'espace théorique ouvert, de ces règles aussi bien que de leur usage.

\section{HISTOIRE OU RÉCIT DE VIE : UNE HISTOIRE DE CAS}

$\underline{\text { Retour à la table des matières }}$

S'il s'agit d'observer un individu ou un groupe, le nouvel espace théorique à construire intéressera le psychologue ou le psychosociologue dont le champ d'observation peut être circonscrit et défini. La question est plus difficile en sociologie : comment en effet observe-ton une société ? C'est sans doute à ce point précis que la démarche clinique s'est révélée la plus féconde pour le sociologue qui ne doit pas moins observer, et ce dans une situation d'implication où l'intimité partagée est la plus radicale, qu'il s'agisse d'étudier sa propre société ou toute autre société. Si les conditions non verbales à partir de quoi le sociologue doit parler ont pu être décrites, la question est désormais la suivante, que nous poserons à la manière de Ramognino (1992) : quelle expérience scientifique le sociologue peut-il envisager sur la base de sa propre expérience sociale ? À défaut de pouvoir observer une société, reconnaissons qu'il est au moins possible d'en observer les

5 Il faudrait une analyse en profondeur de l'ouvrage, pourtant difficile, de Devereux, tellement il est important. Il écrit notamment : « Notre méthodologie [...] doit exploiter la subjectivité inhérente à toute observation en la considérant comme la voie royale vers une objectivité authentique plutôt que fictive. » (Devereux, 1980 : 16.) 
composantes, d'observer plus spécifiquement des cas d'espèce dont la représentativité sociologique peut être établie et répétée. L'étude de cas (Hamel, 1992) est d'abord une méthode clinique par l'observation dont l'espace théorique est maintenant connu. Elle peut être aussi, dans le prolongement de l'approche clinique, une méthode sociologique et tout autant explicative. Mauss l'a fort bien montré :

De plus, c'est une erreur de croire que le crédit auquel a droit une proposition scientifique dépende étroitement du nombre des cas où l'on croit pouvoir la vérifier. Quand un rapport a été établi dans un cas, même unique, mais méthodiquement et minutieusement étudié, la réalité en est autrement certaine que quand, pour la démontrer, on l'illustre de faits nombreux, mais disparates, d'exemples curieux, mais confusément empruntés aux sociétés, aux races, aux civilisations les plus hétérogènes. Stuart Mill dit quelque part qu'une expérience bien faite suffit à démontrer une loi : elle est surtout infiniment plus démonstrative que beaucoup d'expériences mal faites. Or, cette règle de méthode s'applique à la sociologie tout comme aux autres sciences de la nature. (Mauss, 1966 :391.)

L'étude de cas est donc une méthode dont l'usage est à définir dans un espace théorique qui est maintenant connu, mais qu'il reste à préciser suivant la discipline considérée.

L'étude de cas est clinique par la prise en considération de la relation observateur-observé et du statut épistémologique du projet du clinicien dans cette intimité partagée décrite plus haut. Elle est aussi une méthode par le cumul des observations qui lui donne toute sa légitimité. Elle est enfin aussi une méthode par l'analyse des données d'observation recueillies. La difficulté essentielle de l'analyse clinique réside dans le fait que les règles qui régissent la démarche clinique ne sont pas explicites. Elles relèvent d'une démarche qui est avant tout une pratique. Ainsi, quelles sont les règles qui interviennent, par exemple, dans l'art du diagnostic, dans l'observation ou encore dans l'entretien ou l'interview (Poupart, 1993) ? À définir, en effet ; à noter le cumul des cas (Enriquez, 1992) qui sont le fait de tout bon clinicien d'expérience. Ajoutons que si l'espace clinique apparaît d'emblée pluridisciplinaire, il ne présente pas moins des difficultés particulières 
suivant la discipline considérée. C'est bien sûr le cas de la sociologie depuis la collecte des données jusqu'à l'explication proprement dite (Houle, 1993a).

Quelles sont donc ces règles théoriques et méthodologiques à l'œuvre dans l'analyse ? Ou encore, quelles devraient être ces règles ? Deux voies en fait ont été envisagées jusqu'à maintenant. Pour un, Gardin (1991) propose, dans la perspective d'une épistémologie pratique, que l'on analyse les démarches de recherche faites dans le champ des analyses du discours pour en dégager le travail de schématisation scientifique accompli, dès lors observable, et conclure. Cela vaut pour l'usage des histoires de vie et permettrait aussi de conclure. Le constat de Gardin est sévère : ces analyses considérées du point de vue de ce qu'est une démarche scientifique ne satisfont pour ainsi dire à aucun des critères qui définissent la schématisation scientifique. Les sciences sociales seraient de la mauvaise littérature, aussi bien en faire de la bonne, nous suggère-t-il. Bien que les analyses de Gardin n'aient pas porté sur l'usage des histoires de vie en sociologie particulièrement, sa conclusion vaut néanmoins dans l'état actuel des choses.

Reste la deuxième voie, à vrai dire périlleuse : les définir, à proprement parler, ces règles dans la définition de ce que serait le travail du sociologue, c'est-à-dire la construction du savoir sociologique. Pareille prétention est assurément insoutenable. Les conditions sont pourtant réunies qui pourraient le permettre. L'usage des histoires de vie conjugue aux acquis de l'analyse clinique, et considéré du point de vue de ce que serait une méthodologie qui satisfasse aux exigences de rigueur posées précédemment, autorise tout de même quelques conclusions ; nous sommes, en effet, dans la troisième période :

- le sujet est là ;

- son savoir vaut, et ce n'est pas de la sociologie ;

- le sociologue le reconnaît et reconnaît que son travail y trouve ses conditions premières, cliniques ai-je tenté de démontrer ; 
- l'espace théorique ouvert définit les conditions de son travail dans le passage d'une subjectivité, de subjectivités, à une objectivité ;

- il reste à construire cet espace ; il relève de l'étude de cas, base ultime d'observations qui autorise toute généralisation théorique et empirique.

Il est tout à fait possible d'analyser l'usage des histoires de vie dans cette perspective, mais aussi les correspondances ou les journaux intimes. Ce pourrait aussi être le cas des monographies que l'on redécouvre depuis quelque temps en sociologie, sans oublier le journal de terrain (Weber, 1991) comme observatoire privilégié de la relation de l'observateur et de l'observé. Ces différents matériaux sont autant de bases d'observation, d'observatoires de la vie en société. Suivant les modalités particulières de leur construction, ce sont en un mot autant de bases de données dont il faut définir la validité qui autorise le travail de l'analyse et de l'explication (Houle et Ramognino, 1993).

\section{Les histoires de vie comme base de données}

$\underline{\text { Retour à la table des matières }}$

Dans le meilleur des cas, c'est-à-dire si elles sont recueillies dans les meilleures conditions (cliniques), les histoires de vie sont le résultat de la mise en forme d'une expérience ou d'expériences, si l'on veut. Ces expériences de vie, aussi singulières soient-elles, peuvent tout autant être considérées comme expériences de la vie en société. L'histoire ou le récit de vie est un matériau de choix à cet égard, mais ce n'est pas le seul, nous l'avons vu, qui nous donne accès à cette expérience. L'exemple du Polish Peasant demeure une référence essentielle à cet égard. Les histoires de vie étaient en fait complémentaires de l'analyse des correspondances qui constituaient, a-t-on dit avec raison, le matériau parfait, et ce parce qu'elles étaient le fait des immigrants eux-mêmes et de leurs points de vue ; parce que le sociologue n'y était 
pour rien, sans ironie aucune, c'est-à-dire n'a pu exercer quelque influence dans la mise en forme de cette expérience. Ces correspondances et les histoires de vie des immigrants polonais à Chicago sont la mise en forme de l'expérience de l'immigration, de la vie des immigrants aussi bien que de leurs problèmes. Suivant l'objet de recherche défini, ces matériaux sont les composantes d'une base de données, le résultat donc d'une mise en forme dont il reste à définir les modalités. Sur ce chapitre, elles relèvent d'une sociologie de la connaissance.

L'expérience de l'école de Laval est tout aussi intéressante. Après avoir analysé pendant plusieurs années les idéologies et la transformation des idéologies au Canada français, on a voulu analyser ces transformations observées dans le « vécu » des Québécois (Dumont et Gagnon, 1973). À l'occasion d'une immense enquête (survey research), les chercheurs ont recueilli près de 150 histoires de vie pour analyser les «mutations récentes du Québec contemporain » (Gagnon et Jean, 1975), tel que le signalait le titre de la recherche. Les histoires de vie sont la mise en forme de ces transformations comme les ont vécues et racontées les Québécois. Par-delà les histoires singulières, il s'agit ici aussi d'une mise en forme de l'expérience de la vie en société et de ses transformations.

Franco Ferrarotti l'aura écrit à sa manière :

Toute narration autobiographique raconte, selon une coupure horizontale ou verticale, une praxis humaine. Or, «si l'essence de l'homme [...] est, dans sa réalité, l'ensemble des rapports sociaux » (Marx, VI' thèse sur Feuerbach), toute praxis individuelle est activité synthétique, totalisation active de tout un contexte social. Une vie, c'est une praxis qui s'approprie des rapports sociaux (les structures sociales), les intériorise et les retraduit en structures psychologiques par son activité déstructurante-restructurante. Toute vie humaine se révèle jusque dans ses aspects les moins généralisables comme la synthèse verticale d'une histoire sociale. Tout comportement ou acte individuel nous paraît jusque dans ses formes les plus uniques la synthèse horizontale d'une structure sociale. (Ferrarotti, 1979 : $141^{6}$ )

6 Je remercie Isabelle Perrault pour cette traduction. 
Nous dirons plus simplement que s'il y a synthèse de la structure sociale, synthèse des rapports Sociaux, c'est que ces rapports sociaux seraient observables dans les histoires de vie suivant les modalités de cette synthèse, de ce savoir caractéristique des histoires de vie. Il s'agit bel et bien de la mise en forme de l'expérience de la vie en société et ces récits sont la base de données qu'il reste à analyser.

\section{Les histoires de vie comme connaissance}

$\underline{\text { Retour à la table des matières }}$

S'il y a synthèse, c'est bien parce qu'il y a élaboration d'un savoir qui vaut. Par analogie avec le travail théorique, il y a construction d'un objet, de la réalité de l'expérience comme objet et d'après des règles qui sont l'objet d'une sociologie, d'une théorie de la connaissance. Ces règles sont implicites et sont au cœur de la mise en forme de cette connaissance qui relève du sens commun dont la visée n'est pas d'expliquer mais de donner précisément sens à la vie (Houle, 1987). Le sens commun comme forme de connaissance diffère en cela de la connaissance sociologique dont la visée est bien d'expliquer, et ce suivant des règles - théoriques et méthodologiques - qui sont explicites. Ce sont là des niveaux d'objectivation, d'abstraction de la réalité qu'il ne s'agit plus de hiérarchiser, mais bien de différencier en fonction de la relativité des règles qui en définissent la spécificité. À la manière de Granger (1967), précisons encore que, dans le premier cas, il y a « modélisation concrète » de l'expérience donnant un sens immédiat à celle-ci à la différence du modèle abstrait construit par le chercheur aux fins de l'explication recherchée.

Cette piste de recherche est la voie ouverte pour construire théoriquement l'espace décrit plus haut sur la base de conditions non verbales où le sujet est reconnu, le travail du sociologue précisé dans la dif- 
férenciation de savoirs qui établit le statut de l'observateur et de l'observé.

\section{Un modèle concret}

$\underline{\text { Retour à la table des matières }}$

S'il y a modélisation, c'est suivant des règles implicites qui déterminent les modalités de la mise en forme ; le modèle concret est à repérer et à dégager au moment de l'analyse, à repérer explicitement donc. Il faut ici nuancer Ferrarotti. S'il y a synthèse, cette synthèse n'est pas celle des rapports sociaux, mais bien de la vie ou de cette dimension de la vie qui est l'objet de la recherche et des histoires recueillies ; les " rapports sociaux » relèvent du travail théorique et non du sens commun et instruisent clairement la théorie qui permettra virtuellement l'explication. Encore une fois, il faut différencier les niveaux d'objectivation, les formes de connaissance considérées, et ne pas inférer que l'historien de sa vie pense sa vie du point de vue des « rapports sociaux »; c'est là le travail du sociologue.

Ces règles sont repérables dans des discours, elles sont observables dans l'ordre même du langage. Une telle sociologie de la connaissance ressortit méthodologiquement à une sémantique structurale, à l'analyse d'organisations lexicales. Rappelons avec Granger que si une forme de connaissance est relative à l'objet dont elle est l'expression et aux règles de sa construction, ces règles renvoient aux points de vue de l'usage linguistique, lesquels «correspondent à une orientation de la structuration dans son rapport à une expérience » (Granger, 1968 : 172). Si structuration de l'expérience il y a dans le langage, cette structuration est bel et bien observable dans une forme de connaissance : ce sont précisément les modalités de cette structuration, aussi bien que sa visée, qui permettent de définir des niveaux d'abstraction, des types d'objectivité qu'il ne s'agit plus de hiérarchiser ; il s'agit plutôt d'apprécier la relativité de ces savoirs constitutifs les uns des autres, dans 
l'usage diversifié du langage. Il y a donc modélisation concrète de l'expérience dans le langage, dans une organisation lexicale dont la structuration est relative à une orientation de la pratique.

Voilà ce qui autorise l'usage des histoires de vie ou des correspondances, ce sont là des discours relevant du sens commun défini comme forme de connaissance. L'étude du cas des immigrants polonais vivant à Chicago, du point de vue de l'immigration, est possible en effet ; cette étude aurait pu être faite aussi du point de vue de la famille, du travail ou de la santé. Il en est de même du cas des Québécois, de la société québécoise, du point de vue des mutations en cours ; ou d'autres points du vue encore, telles leur économie ou leur vie politique. Ce point de vue est fondamental, c'est celui de l'objet de la démarche de recherche à l'origine du recueil des histoires de vie, de cette mise en forme dans la construction d'une base de données (Hurtubise, 1991) ; à l'origine aussi du savoir sociologique qui pourra expliquer une pratique du point de vue de cette orientation, c'est-à-dire de cet objet venant découper cette pratique, d'un découpage fait dans le respect de ces conditions non verbales décrites par Foucault.

\section{De l'analyse}

$\underline{\text { Retour à la table des matières }}$

Dans une telle perspective, les règles à l'oeuvre sont des règles sociales. Le sens commun est une forme de connaissance dont la logique renvoie aux modalités de cette mise en forme, c'est-à-dire à la relativité des rapports sociaux dont les propriétés sont alors saisissables à travers la construction qui en est faite comme forme sociale, suivant les règles donc qui sont la construction de cette logique sociale. Nous touchons là, et du point de vue particulier du sens commun, ce que dès le début des années 20 Lukács définissait comme "le système des formes d'objectivité de cette vie réelle » (Lukács, 1923 : 238), envisagé ici du point de vue de cette forme particulière d'objectivité que vise 
la sociologie de la connaissance. Si ces règles relèvent d'une " praxis culturelle ", à la manière même dont Gagnon (1980) en a proposé la théorie, force est de reconnaître qu'il n'y a précisément de praxis culturelle que parce qu'il y a praxis, saisissable dans l'« expérience » ou dans les diverses formes d'expression discursive qui sont autant de modes d'accès à cette expérience, aux rapports sociaux, écrira Ferrarotti.

Quel est donc le travail d'analyse dès lors possible à propos de cette pratique dont nous connaissons maintenant les conditions ? Posons de manière trop brève et trop schématique ce que toute analyse exige :

1) l'observation - la construction d'un observatoire, d'une base de données - du phénomène à l'étude ; ou encore, le recueil d'histoires ou de récits de vie, la recherche d'une correspondance ;

2) la description de cette base de données. En bref, de quoi parle-ton et en quels termes ? quels sont les lexiques en oeuvre et quelles relations lexicales peut-on observer à propos du phénomène à l'étude ? Il s'agit de décrire ici l'histoire ou le récit de vie en vertu des diverses organisations lexicales repérables ;

3) une réduction en fonction des relations lexicales repérées. Suivant les observations faites, quelles seraient les relations lexicales au principe de structuration des organisations lexicales observées, dans leur rapport avec l'expérience, c'est-à-dire avec le phénomène à l'origine de la recherche ? Par confrontation heuristique des données pourvues de sens, par quelles relations sémantiques serait mis en forme l'objet de la recherche dans le langage ? Il s'agit ici de définir, en toute hypothèse, ce que serait la modélisation concrète observée, objet d'analyse, objet virtuel d'une explication ; 
4) l'explication doit permettre d'établir en quoi, comment et pourquoi cette logique sémantique est aussi une logique sociale, en quoi le modèle concret vient « donner unité à l'expérience » qui s'y trouve construite.

Le passage de la description à l'analyse est indispensable à l'explication. Ces deux moments de la recherche, il est important de les distinguer, ne serait-ce que pour démontrer :

- le caractère indépassable de l'interprétation en tant que partie intégrante de toute démarche scientifique ;

- le caractère essentiel de toute théorie dans le travail d'analyse.

Les règles descriptives sont à la base de tout travail scientifique, alors que les règles explicatives se rattachent à l'objet d'analyse de toute science, qu'elle soit sociale ou non. Cette distinction est fondamentale : les règles secondes (explicatives) ne peuvent que suivre les premières (descriptives). Leur confusion ou leur conjugaison dans le même temps est à l'origine des divers positivismes en sociologie. Ferrarotti, dans son ouvrage sur « Ia sociologie à la redécouverte de la qualité », a fort bien posé le problème :

À l'ancienne idée positiviste naïve suivant laquelle il suffisait d'observer pour obtenir des données et que, par conséquent, l'observation était un processus naturel qu'il n'était pas besoin de situer et de pondérer selon des points de vue particuliers ou des « équations personnelles » étant donné que chaque acte d'observation était d'emblée un acte d'interprétation, il s'est substitué la conscience qu'entre la réalité du monde et le langage, il n'y a pas un simple «effet de miroir » et qu'il ne suffit pas d'observer, y compris de manière critique, pour transformer une donnée pragmatique, ou considérée comme fait, en donnée problématique. (Ferrarotti, 1989 : $42{ }^{7}$ )

7 Je remercie Isabelle Perrault pour cette traduction. 
Il ne s'agit pas ici de dissoudre l'objet de la sociologie dans la description, mais de reconnaître qu'il doit s'y fonder. La construction de l'objet du sociologue trouve dans la description sa limite et ses possibilités ${ }^{8}$. Toute donnée exige d'abord sa déconstruction empirique, c'est-à-dire sa description dans l'ordre même de ce qu'elle est. Ces données sont déjà connaissance, savoir qui recèle l'objet empirique à rechercher, déterminant de toute explication. Car celle-ci n'est rien d'autre que la construction théorique des propriétés de cet objet dégagé de sa forme première de connaissance, du point de vue d'une forme seconde à construire. Allons plus loin. Il ne s'agit plus de savoir s'il faut décrire ou pas. Cette forme première de connaissance relève d'une définition, d'une théorie, d'une sociologie de la connaissance dont c'est l'objet. Cette sociologie de la connaissance représente alors le passage obligé de toute sociologie et pourrait rejoindre ce que serait éventuellement une épistémologie de ce savoir premier dont la visée serait ici sociologique, mais qui peut être aussi psychologique, historique ou autre. À la base donc de tout projet théorique. D'une manière plus générale, la définition opératoire de la relation entre théorie et méthode aussi bien que le statut épistémologique de la démarche de construction des données renvoient au problème de ce que serait une méthodologie générale en sciences sociales et en sociologie particulièrement.

\section{CONCLUSION}

$\underline{\text { Retour à la table des matières }}$

Les histoires de vie auront permis de mettre la sociologie face à elle-même : en quoi les explications fournies par le sociologue sontelles plus satisfaisantes que celles de l'homme de la rue ? L'histoire de la sociologie fait voir qu'elle s'est édifiée pour l'essentiel sur la base de

8 C'est dans cette perspective que nous avons tenté d'expliquer la dénatalité au Québec (Houle et Hurtubise, 1991). 
la disqualification du sens commun. Elle s'est par ailleurs engouffrée dans des impasses telles que le savoir élaboré offre un intérêt plus technique (Habermas, 1976: 225-247) que théorique (Berthelot, 1996). Comme l'a déjà souligné Enriquez avec ironie, l'État, à la manière de l'ivrogne qui s'appuie sur un réverbère, a besoin des sciences sociales, moins pour voir clair que pour se tenir debout. Ses nouveaux concurrents sont d'ailleurs les managers, les tenants de la gestion des ressources humaines, comme si l'on pouvait gérer la vie en société.

La partie n'est pourtant pas perdue. C'est le retour non pas du sujet, mais du refoulé ; le sujet est là, il parle et il sait bien parler tant de luimême que de la société au sein de laquelle il vit. Peut-être faut-il d'ailleurs rappeler que, par-delà les chiffres et les lettres, la vie en société est l'objet premier et dernier de la sociologie, qu'il n'y a de société et de vie en société qu'à partir du moment où cela fait sens. Que ce sens enfin est au centre du processus de constitution de toute société ; le disqualifier ne revient à rien d'autre que de disqualifier l'objet même de la discipline. Le sujet est là, saura-t-on l'écouter, lui poser les bonnes questions ? La question est là aussi et l'apport de l'approche clinique aura été déterminant : le sujet est là, sa parole vaut ; il est possible alors d'apprécier le potentiel heuristique considérable que recèlent les histoires ou récits de vie, pour la sociologie et les sciences sociales en général.

Il y a construction du réel dans le sens qui lui est donné, construction de la vie en société dans le sens qui lui est aussi donné. On peut donc l'observer suivant les diverses modalités par lesquelles il y a mise en forme de ce contenu dans l'élaboration des savoirs les plus divers. On a beau « être l'ami des formes, l'on reste fils de la terre ». La question est complexe, et les enjeux « approchés », comme je l'ai posé au point de départ, exigeront bien, en effet, toutes les ressources du « front commun des travailleurs de la preuve ». 


\section{Bibliographie}

$\underline{\text { Retour à la table des matières }}$

ARDOINO, J. (1989). « De la clinique », Réseaux, no 55-57.

BACHELARD, G. (1949). Le rationalisme appliqué, Paris, PUF.

BACHELARD, G. (1968). Essai sur la connaissance approchée, Paris, Vrin.

BATESON, M.-C. (1989). Regards sur mes parents, Paris, Seuil.

BERTAUX, D. (sous la dir. de), (1980). « Histoires de vie et vie sociale », Cahiers internationaux de sociologie, vol. 69.

BERTAUX, D. (sous la dir. de), (1981). Biography and Society, The Life History Approach in the Social Sciences, Londres, Sage.

BERTHELOT, J.-M. (1996). Les vertus de l'incertitude. Le travail de l'analyse en sciences sociales, Paris, PUF.

BRUNET, M., et GAGNON, S. (1993). Discours et pratiques de l'intime, Québec, Institut québécois de recherche sur la culture.

DE GAULEJAC, V., et Roy, S. (sous la dir. de), (1993). Sociologies cliniques, Paris, Éditions Épi, coll. « Hommes et perspectives ». 
DEVEREUX, G. (1980). De l'angoisse à la méthode dans les sciences du comportement, Paris, Aubier.

DUMONT, F., et GAGNON, N. (sous la dir. de), (1993). « Le vécu », Recherches sociographiques, vol. 14, no 2.

ENRIQUEZ, E. (1992). L'organisation en analyse, Paris, PUF.

ENRIQUEZ, E., HOULE, G., RHÉAUME, J., et SÉVIGNY, R. (sous la dir. de), (1993). L'analyse clinique dans les sciences humaines, Montréal, Éditions Saint-Martin.

FERRAROTTI, F. (1979). "Sur l'autonomie de la méthode biographique », dans J. Duvignaud (sous la dir. de), Sociologie de la connaissance, Paris, Payot.

FERRAROTTI, F. (1989). La sociologie alla riscoperta della qualita, Roma-Bari, Editori Laterze, Sagittari 21.

FOUCAULT, M. (1972). Naissance de la clinique, Paris, PUF.

GAGNON, N. (1980). «Données autobiographiques et praxis culturelle », Cahiers internationaux de sociologie, vol. 69, p. 291305.

GAGNON, N., et JEAN, B. (1975). « Les histoires de vie et la transformation du Québec contemporain », Sound Héritage, vol. 4, no 1, p. 55-63.

GARDIN, J.-C. (1991). Le calcul et la raison. Essai sur la formalisation du discours savant. Paris, Éditions de l'École des hautes études en sciences sociales.

GIDDENS, A. (1987). La constitution de la société, Paris, PUF. 
GRANGER, G.-G. (1967). " Science, philosophie et idéologie », Uit. Tijdschrift voor filosofie, 29, Jaargang, 4, December, p. 775776.

GRANGER, G.-G. (1968). Essai d'une philosophie du style, Paris, Armand Colin.

GUSDORF, G. (1990). Lignes de vie, tome 1 : Les écritures du moi ; tome 2 : Auto-bio-graphie, Paris, Odile Jacob.

HABERMAS, J. (1976). Connaissance et intérêt ; Paris, Gallimard.

HAMEL, J. (sous la dir. de), (1992). « La méthode de cas en sociologie », Current Sociology, vol. 40, no 1, printemps.

HOULE, G. (1982). «Présentation. La sociologie : une question de méthodes », Sociologie et sociétés, vol. 14, no 1, p. 3-6.

HOULE, G. (1986). « Histoires et récits de vie : la redécouverte obligée du sens commun », dans D. Desmarais et P. Grell (sous la dir. de), Les récits de vie : théorie, méthode et trajectoires types, Montréal, Éditions SaintMartin, p. 35-51.

HOULE, G. (1987). «Le sens commun comme forme de connaissance : de l'analyse clinique en sociologie », dans Sociologie et sociétés, vol. 19, no 2, p. 77-87. [Texte disponible dans Les Classiques des sciences sociales. JMT.]

HOULE, G. (1993a). "L'analyse clinique en sciences humaines », dans E. Enriquez, G. Houle, J. Rhéaume et R. Sévigny (sous la dir. de), L'analyse clinique dans les sciences humaines, Montréal, Éditions Saint-Martin, p. 39-53.

HOULE, G. (1993b). « Pour une sociologie de la connaissance de la vie : de l'usage des histoires de vie », dans $\mathrm{M}$. Brunet et S. 
Gagnon (sous la dir. de), Discours et pratiques de l'intime, Québec, Institut québécois de recherche sur la culture, p. 229-245.

HOULE, G., et HURTUBISE, R. (1991). « Parler de faire des enfants, une question vitale », Recherches sociographiques, vol. 32, no 3, p. 385-415.

HOULE, G., et RAMOGNINO, N. (sous la dir. de), (1993). « La construction des données », Sociologie et sociétés, vol. 25, no 2, automne.

HURTUBISE, R. (1991). L'amour, le soi et la société. Sociologie de la connaissance amoureuse dans les correspondances québécoises (1860-1988), thèse de doctorat, Université de Montréal.

LUKÁCS, G. (1923). Histoire et conscience de classe (1960), Paris, Minuit.

MAUSS, M. (1966). Sociologie et anthropologie, Paris, PUF. [Texte disponible dans Les Classiques des sciences sociales. JMT.]

MEAD, G.H. (1934). L'esprit, le soi et la société (1963), Paris, PUF.

PIRES, A.P. (1982). « La méthode qualitative en Amérique du Nord. un débat manqué (1918-1960) », Sociologie et sociétés, vol. 14, no 1, p. 15-29.

PIRES, A.P. (1994). « La recherche qualitative et le problème de sa scientificité », Cahier de recherche no 9401C, département de criminologie, faculté des sciences sociales, Université d'Ottawa. 
POUPART, J. (1993). « Discours et débat autour de la scientificité des entretiens de recherche », Sociologie et sociétés, vol. 25, no 2, p. 93-110.

RAMOGNINO, N. (1992). «L'observation, un résumé de la réalité », Current Sociology, vol. 40, no 1, printemps, p. 55-75.

SIMEONI, D., et DIANI, M. (1995). « Biographical Research », Current Sociology, vol. 43, nos 2-3, p. 221-258.

THOMAS, W., et ZNANIECKi, F. (1958). The Polish Peasant in Europe and America, New York, Dover Publications, Inc., 2 vol.

THOMPSON, P. (1978). The Voice of the Past, Oral History, Oxford, Oxford University Press.

TOURAINE, A. (1984). Le retour de l'acteur, Paris, Fayard.

WEBER, F. (1991). «L'enquête, la recherche et l'intime ou : pourquoi censurer son journal de terrain ? », Espaces Temps/Les Cahiers, nos 47-48, p. 71-81.

WIRTH, L. (1931). « Chnical Sociology », American Journal of Sociology, vol. 37, no 1, p. 49-66.

Fin du texte 